\title{
Uma Aplicação da Realidade Aumentada no Ensino de Modelagem dos Sistemas Estruturais
}

\author{
Claudia Susie Camargo Rodrigues ${ }^{1}$ \\ Ricardo Alexandre Marquezin Pinto ${ }^{2}$ \\ Paulo Fernando Neves Rodrigues ${ }^{2}$
}

\begin{abstract}
Resumo: Muitos estudos mostram que os estudantes aprendem mais quando diversas técnicas de ensino são utilizadas e que alguns alunos respondem melhor a determinada metodologia de ensino. Partindo desse pressuposto, o uso dos computadores em sala de aula foi cada vez mais incentivado e diversas aplicações de técnicas computacionais foram desenvolvidas como ferramentas educacionais, com o objetivo de proporcionar aos alunos um vasto campo de experiências de aprendizagem. Uma das maiores dificuldades, em termos pedagógicos, nos cursos de arquitetura é a interface entre as disciplinas de estruturas e as demais disciplinas de projeto arquitetônico. Este artigo apresenta uma aplicação da Realidade Aumentada implementada uma ferramenta de ensino na Faculdade de Arquitetura e Urbanismo da Universidade Federal do Rio de Janeiro (UFRJ), com o intuito de introduzir os alunos no estudo dos sistemas estruturais, de uma maneira lúdica e adequada ao perfil dos estudantes de Arquitetura.
\end{abstract}

Palavras-chave: Realidade Aumentada. Modelagem de sistemas estruturais. Análise qualitativa.

\begin{abstract}
Many studies have shown that students learn best when a variety of teaching techniques are used, and that some students respond best to certain methods. Based on this, the use of computers in classroom is more and more stimulated and many applications of computational techniques are being developed as educational tools, with the aim of providing a vast field of learning experiences for the students. One of the most difficult pedagogical problems in architectural education is the interface between structural and architectural design issues. This paper presents an Augmented Reality application implemented as a teaching tool in the Faculty of Architecture and Urbanism at Federal University of Rio de Janeiro (UFRJ), to introduce the novice students to the study of structural systems, in a pleasant manner suitable for the architecture student profile.
\end{abstract}

Keywords: Augmented Reality. Modeling of structural systems. Qualitative analysis.

\section{Introdução}

O estudo de estruturas, na formação acadêmica dos estudantes de arquitetura, é considerado uma tarefa difícil por muitos e, até mesmo, enfadonha por alguns, devido ao uso do instrumental matemático necessário à apresentação de determinados conceitos da engenharia. Particularmente, tirando-se partido da privilegiada capacidade de percepção visual dos alunos de Arquitetura, é possível facilitar a compreensão do comportamento dos elementos estruturais básicos no seu primeiro contato com esse assunto. Esse objetivo pode ser alcançado

\footnotetext{
${ }^{1}$ COPPE/Universidade Federal do Rio de Janeiro, Lab3D - Laboratório de Realidade Virtual, Centro de Tecnologia, Bloco I

- Sala 044a - Cidade Universitária - 21949-900 - Rio de Janeiro (RJ) - Brasil

\{susiedcos.ufrj.br\}

${ }^{2}$ FAU/Universidade Federal do Rio de Janeiro, Departamento de Estruturas, Av. Pedro Calmon, 550 - Prédio da FAU/Reitoria Sala 320 - Cidade Universitária - 21941-901 - Rio de Janeiro (RJ) - Brasil

\{pfnr@fau.ufrj.br, marquezin@ufrj.br\}
}

doi: $10.5335 /$ rbca. 2010.017

Revista Brasileira de Computação Aplicada (ISSN 2176-6649), Passo Fundo, v.2, n. 2, p. 81-95, set. 201081 
pelo estudo das propriedades dos sistemas estruturais através de uma análise qualitativa, principalmente pela observação das configurações deformadas de modelos reduzidos. Neste tipo de análise, os conceitos básicos dos elementos estruturais são estudados através de uma abordagem não matemática. Muitos trabalhos foram escritos, nesse contexto, no campo da educação, podendo-se citar, entre outros, os de Salvadori e Heller [1], Polillo [2], Gordon [3], Salvadori e Tempel [4], Santos [5] e Hilson [6].

A análise qualitativa feita com o uso de modelos físicos reduzidos construídos com materiais relativamente flexíveis, como borracha, silicone, papelão e isopor foi apresentada, inicialmente, por Salvadori e Tempel [4]. A aplicação desses modelos qualitativos com o intuito de simular as configurações deformadas de elementos estruturais, impostas pela ação de cargas nos mesmos, proporciona uma maneira fácil e eficiente de se entenderem os conceitos básicos da engenharia, tais como tração, compressão, flexão, flambagem e torção, despertando o sentimento e a intuição dos alunos. Além disso, este procedimento pode ajudar no processo de integração das disciplinas de estruturas e de projeto de Arquitetura, que se constitui numa das grandes dificuldades encontradas por muitos professores nos primeiros períodos letivos do curso de arquitetura [7,8].

A evolução da tecnologia multimídia possibilitou o desenvolvimento de técnicas computacionais voltadas para a interação com o ambiente de aprendizagem. A aplicação dessas ferramentas constitui um passo importante para o preenchimento das lacunas existentes na formação dos estudantes de arquitetura.

Em algum momento, num programa de televisão, num noticiário ou num filme de ficção científica, o espectador deve ter visto uma pessoa de capacete experimentando sensações de um mundo simulado artificialmente pelo computador. Esta tecnologia que tenta recriar ao máximo a sensação da realidade para um indivíduo através de interações, em tempo real, com o uso de técnicas e de equipamentos computacionais é chamada de Realidade Virtual (RV). Exemplos de aplicação da RV são apresentadas em diversos jogos para computador, como os que simulam jogos de futebol.

$\mathrm{Na}$ área de educação, diversos trabalhos foram publicados para o ensino de estruturas, aplicando algumas técnicas de Realidade Virtual (RV), nas últimas duas décadas [9, 10,11, 12, 13]. Porém, esses possuem características bastante distintas, não possibilitando uma comparação direta entre os mesmos.

A Realidade Aumentada (RA), por sua vez, tem como base o mundo real, colocando sobre ele informações ou objetos virtuais; pode ser reconhecida em jogos de futebol exibidos pela televisão, quando uma seta marca a distância entre a bola e o gol, ou quando uma circunferência determina a separação entre a bola e a barreira de jogadores adversários numa cobrança de falta.

Este artigo apresenta uma aplicação de RA para incrementar o conhecimento do comportamento estrutural por meio de modelos físicos qualitativos. Esta ferramenta foi desenvolvida para ser aplicada, principalmente, no ensino de estruturas para os alunos calouros da Faculdade de Arquitetura e Urbanismo da Universidade Federal do Rio de Janeiro (FAU/UFRJ). O restante deste artigo está organizado de forma que a seção 2 descreve, brevemente, a metodologia empregada na disciplina "Modelagem dos Sistemas Estruturais" [14] do primeiro período letivo do curso de Arquitetura e Urbanismo da UFRJ; na seção 3, algumas características da RA são descritas sucintamente e, na seção 4, a aplicação desenvolvida neste trabalho é apresentada em detalhes; por fim, conclusões e sugestões para trabalhos futuros são formuladas.

\section{Modelagem dos Sistemas Estruturais}

O estudo dos sistemas estruturais é essencial na formação acadêmica dos estudantes de arquitetura. Na FAU/UFRJ, a introdução deste assunto ocorre numa disciplina sobre modelagem de estruturas para a simulação e teste de modelos físicos, que tem se mostrado como uma abordagem que em muito contribui para o entendimento dos conceitos básicos da Engenharia e do projeto arquitetônico. Neste estágio, as características e propriedades dos elementos estruturais são extensivamente estudadas. As principais propostas são: a) permitir aos alunos uma aquisição da sensibilidade necessária ao entendimento dos diversos sistemas estruturais; b) motivá-los, despertando o interesse pelo estudo da análise estrutural nos períodos letivos subsequentes. A compreensão mais completa possível dos conceitos básicos de estruturas é considerada um pré-requisito para a introdução aos módulos seguintes.

A metodologia de ensino baseia-se em exposições teóricas sobre diversos sistemas estruturais e seus elementos, com apresentação de imagens, seguidas de contato direto com modelos didáticos [14]. Em seguida, os alunos desenvolvem projetos de sistemas estruturais, inspirados na obra de determinados arquitetos; constroem 
seus próprios modelos reduzidos (Figura 1), utilizando materiais apropriados para o estudo, e analisam qualitativamente o comportamento estrutural dos mesmos. Esta análise tem como objetivo adquirir uma certa sensibilidade ao estudo de equilíbrio, tensões e deformações causadas por esforços de tração, compressão, flexão e torção, associadas aos elementos que compõem o seu projeto.

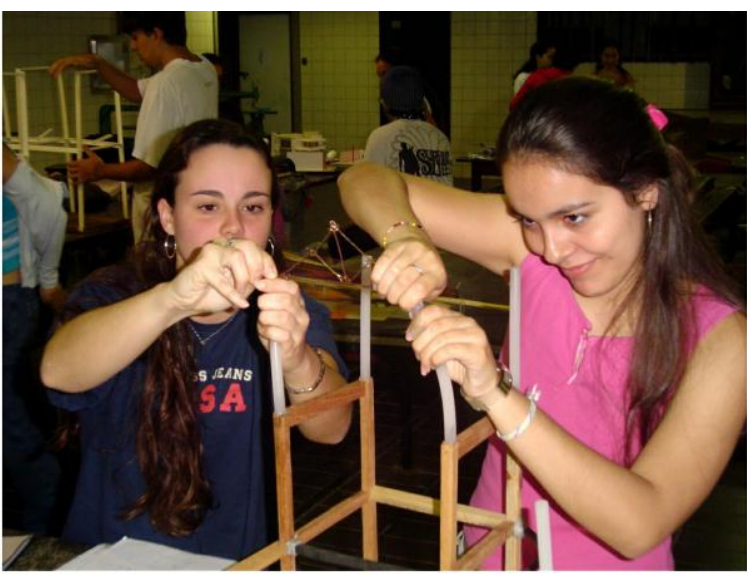

(a)

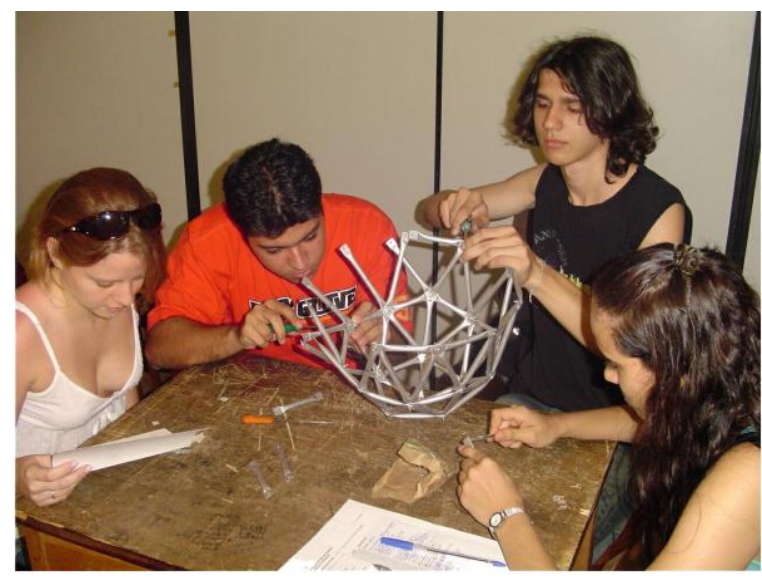

(b)

Figura 1. Estudantes construindo modelos qualitativos em sala de aula

A metodologia aplicada no curso inclui o emprego da intuição no processo de aprendizagem através de exemplos de estruturas encontradas na natureza, da observação dos sucessos e falhas das estruturas do passado e do presente e, inevitavelmente, das respostas de modelos físicos qualitativos a determinadas ações. De acordo com a ementa da disciplina, os alunos são obrigados a construir os modelos físicos e a interagir com os mesmos, principalmente pela manipulação e observação (Figura 2).

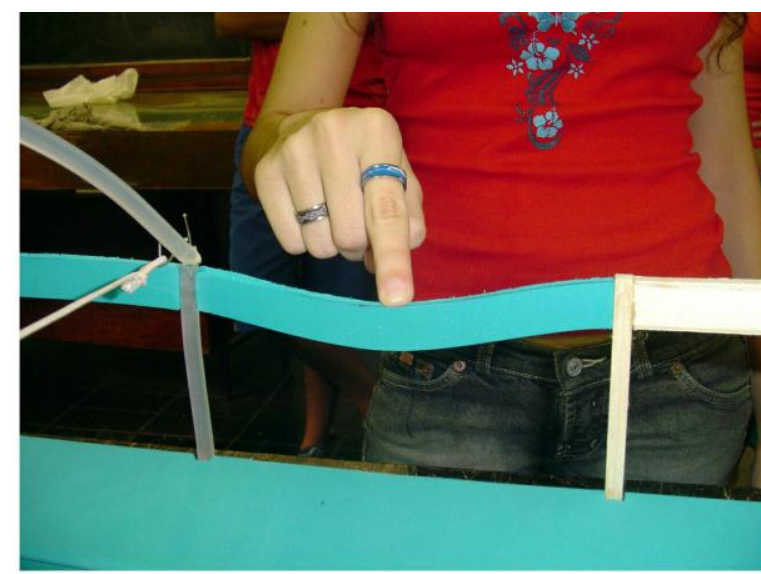

(a)

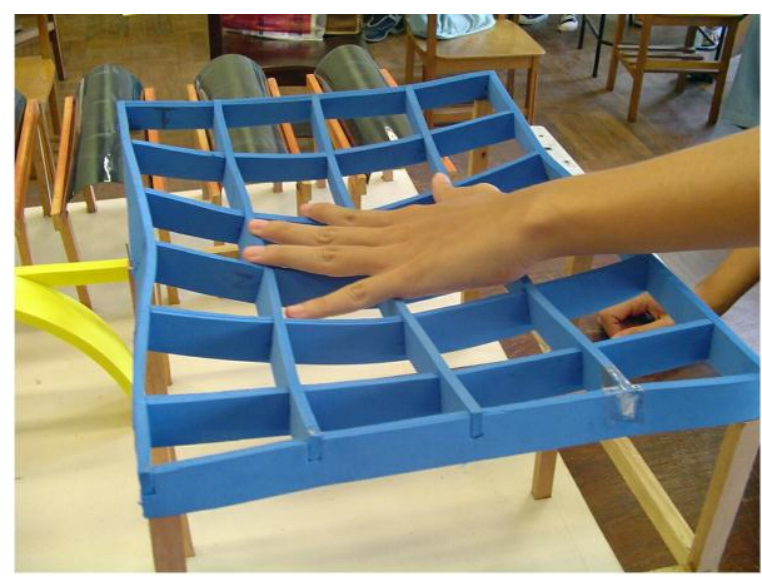

(b)

Figura 2. Estudantes manipulando modelos

A intenção é mostrar aos alunos a funcionalidade da estrutura no projeto de arquitetura, sua relevância nos processos de projeto e construção, introduzindo o conhecimento dos sistemas estruturais de forma qualitativa e lúdica, incluindo suas propriedades e comportamento quando submetidos a certos carregamentos. Esse procedimento possibilita uma melhor compreensão dos conceitos básicos dos sistemas estruturais, tirando vantagem da percepção visual dos estudantes de arquitetura. Desse modo, o assunto torna-se mais atraente para os alunos e compõe uma base para a análise estrutural, sem ser superficial. 


\section{Realidade Aumentada}

A Realidade Aumentada (RA) é uma tecnologia nova e emergente, uma evolução da RV. Ela suplementa o mundo real com objetos virtuais que parecem coexistir no mesmo espaço, por meio de algum dispositivo tecnológico $[15,16]$. A RA pode adicionar gráficos, sons, tato e cheiro ao mundo natural. Como o nome já diz, a RA "aumenta" o ambiente para o usuário, sendo capaz de ampliar sua percepção e a sua interação com o mundo real. Ela enriquece este ambiente com informações virtuais que ajudam no desempenho de suas tarefas. Azuma et al. [15] definiram algumas características de um sistema para que seja considerado de RA: "combinar objetos reais e virtuais num ambiente real", "operar interativamente, e em tempo real" e "registrar (alinhar) objetos reais e virtuais, uns com os outros".

\subsection{Dispositivos utilizados}

A interação é fundamental em sistemas de RV e RA e deve dar ao usuário a sensação de presença no ambiente virtual. Dependendo dos estímulos providos por este ambiente em tempo real, a reação do usuário pode ser diferente. São permitidas a este a movimentação e a manipulação de objetos. Para proporcionar essas sensações, é necessária uma interface entre o usuário e o ambiente virtual que deve ser capaz de identificar estes estímulos, passá-los para a forma de sinais digitais e fornecer uma resposta perceptível para o usuário.

A seguir, são apresentados os principais dispositivos utilizados tanto na RV quanto na RA que oferecem maneiras mais intuitivas de interação ou que aumentam o nível de imersão do usuário. É importante observar que, a cada dia, novos dispositivos são constantemente desenvolvidos, com o objetivo de oferecer modos mais intuitivos de interação. E a partir de 2000, ficaram mais acessíveis, pelos melhores índices de custo-benefício.

\subsubsection{Sistemas de Exibição}

Os equipamentos de visualização, como os HMD (Head-Mounted Displays), são tecnologias tradicionais de saída para aplicações de RV e RA. O Head-Mounted Display (HMD) baseado em vídeo não permite visão direta do mundo real e é mais usado em RV, fornecendo imagens reais por meio de câmeras de vídeo acopladas no exterior deste equipamento. Na RA, a imagem projetada ao usuário é mostrada em tempo real e a imagem virtual é sobreposta ao vídeo.

Os HMDs com tecnologia óptica são capacetes ou óculos de RV para apresentar o ambiente virtual diretamente sobre o mundo real em sistemas de RA; diferentes dos HMDs baseados em vídeo, permitem uma visão direta do mundo real; funcionam com uma ou duas câmeras posicionadas na cabeça do usuário, provendo as imagens do mundo real. O vídeo que advém dessas câmeras é, então, combinado com as imagens gráficas que foram criadas pelo gerador de cenas, combinando, assim, real e virtual. Podem ser utilizados em aplicações nas quais se necessita de uma visão ampla, como, por exemplo, em simuladores de torre de controle de tráfego aéreo e em aplicações como o sistema de cirurgia guiada do MIT, focado em cirurgia cerebral [17].

O Sistema Baseado em Monitor pode utilizar monitores convencionais de computador (Desktop) ou portáteis (Palm ou Pocket PC) como dispositivos de apresentação. Esse tipo de esquema é o mais simples de ser construído e não fornece um alto nível de imersão, pois em sua configuração mais convencional exige apenas um monitor e uma câmera como equipamentos. As imagens capturadas pela câmera são alinhadas aos objetos virtuais.

A estereoscopia acrescenta a dimensão de profundidade às telas de projeção dos mundos virtuais, tornando-os mais realistas, mais próximos à forma como os usuários os veem no mundo real. A visualização de imagens 3D é obtida por meio de duas imagens diferentes que são geradas, uma para o olho esquerdo e outra para o olho direito. Existe um ângulo entre essas imagens, gerado por uma defasagem que varia em torno de 6,5 $\mathrm{cm}$, equivalente à distância que separa os olhos humanos. Há várias técnicas de estereoscopia, entre elas, a Técnica de Polarização da Luz. Nesta técnica, dois projetores comuns são usados para projetar imagens diferentes para o olho esquerdo e para o direito. Filtros de luz com polarização oposta são colocados sobre a lente de cada projetor. Cada usuário deve usar óculos com as lentes polarizadas de forma correspondente, de modo que cada olho enxergue somente a imagem correta. Uma tela de projeção prateada (ou aluminizada) é necessária para preservar a polarização da luz [18]. 
O dispositivo de projeção de imagens diretamente na retina ou Dispositivo Virtual de Retina (VRD) é um sistema de exibição mais interessante e simples do ponto de vista do usuário. Ele utiliza varreduras moduladas com feixes de laser de baixa potência para criar a percepção da imagem virtual, que é desenhada diretamente na retina do usuário. O modo pelo qual se enxerga é o seguinte: um laser de diodo vermelho provê um display monocromático. Um laser de gás argônio produz linhas verdes e azuis. E os dois combinados proveem a criação de um display full color [19].

\subsubsection{Dispositivos Hápticos}

Um dispositivo háptico é aquele que envolve sensação tátil ao usuário quando em contato com objetos virtuais. Estes dispositivos podem ser usados por pessoas com deficiências ou pessoas que aprendem melhor através de experiências táteis ou sinestésicas [19].

Como exemplo de dispositivos hápticos podem-se citar as luvas de dados (data gloves), as quais são equipadas com sensores que sentem os movimentos dos dedos da mão e fazem a interface desses movimentos com o computador, permitindo ao usuário manipular objetos ou realizar movimentos em um ambiente virtual.

\subsubsection{Dispositivos Móveis}

São dispositivos pequenos e mais ergonômicos, como celulares e computadores de mão, como Pocket PC, tablet PC e Personal Digital Assistant (PDA). Esses dispositivos têm uma boa relação entre poder de processamento, tamanho e peso, além de serem fáceis de manipular e possuírem conectividade sem fios. Outra opção é a não utilização de dispositivo, ou seja, é o uso da própria mão. Estudos incentivam o uso deste tipo de interação (via gestos) muito mais intuitivo nos ambientes virtuais. E ainda, os usuários com necessidades especiais podem se beneficiar com este tipo de manipulação. A mão é gravada por uma câmera de vídeo e digitalizada por um hardware especial. Um software de reconhecimento de imagem usa os dados da imagem para determinar a posição atual e a forma (gesto) da mão. Esta informação é então utilizada para conduzir a aplicação [20].

\section{2 Áreas de aplicações}

É imenso o potencial da RV e da RA e, nesta seção, são mostrados alguns exemplos de áreas de aplicações.

\subsubsection{Manutenção}

Diversas pesquisas [15] têm sido feitas para o desenvolvimento de aplicações para ajudar em reparos e manutenções de um modo geral. Para facilitar o entendimento das instruções, ao invés de ler manuais e observar figuras, objetos 3D podem ser sobrepostos a um equipamento qualquer, mostrando passo a passo as tarefas que devem ser feitas e como fazê-las. Estes objetos podem ainda ser animados para que os passos sejam mostrados de uma maneira mais explícita. Algumas aplicações, já existentes, consistem na manutenção de uma impressora a laser e, ainda, na manutenção de um encanamento industrial, onde são visualizados um mapa 2D e um modelo $3 \mathrm{D}$.

\subsubsection{Militar}

Desde os primeiros momentos na história da RV, as forças do exército dos Estados Unidos foram um fator importante no desenvolvimento e na aplicação de novas tecnologias de RV. Junto com as áreas médica e de entretenimento, a militar é responsável pela evolução mais rigorosa no campo da RV. Os simuladores de voo são as aplicações mais utilizadas, treinando os soldados para voar em batalha, se restabelecer em caso de emergência ou coordenar a sustentação no ar com operações terrestres. Os simuladores de veículos terrestres, não tão precisos quanto os simuladores de voo, são uma parte importante da estratégia do exército. Existem ainda cenários virtuais realistas de imersão que ajudam os soldados a se adaptarem a vários ambientes e situações de combate, além da visualização do campo de batalha, essencial ao determinar as estratégias de combate em tempo real [15]. 


\subsubsection{Visualização e Explicação}

Uma aplicação muito usada em RA consiste em colocar pequenas notas em objetos e ambientes, as quais contêm informações públicas e particulares. $O$ interessante nesse tipo de aplicação é a ajuda em tarefas cotidianas, como guiar o usuário através de um prédio desconhecido, mostrando uma variedade de sugestões para a navegação, ou como guiá-lo através de um museu, mostrando várias informações à medida que o usuário caminha.

Sistemas de RV e RA oferecem inúmeros recursos para a visualização de informações de um grande volume de dados, uma vez que não há limite de espaço para exploração dos dados a serem representados.

\subsubsection{Entretenimento}

As aplicações na área de entretenimento são as mais diversas possíveis, desde jogos até "contadores" de histórias. Entre os jogos criados com RA, pode-se destacar o ARQuake [21]. Este é jogado no mundo real, dando ao usuário a capacidade de se mover por onde desejar. Tudo que é visto é determinado exclusivamente pela orientação e posição da cabeça do usuário que está usando um HMD.

Outra aplicação que mistura entretenimento e aplicação móvel é o Invisible Train [22]. Esse jogo foi desenvolvido, primeiramente, para crianças de nível primário e é um jogo multiusuário no qual os jogadores guiam um trem virtual através de um trilho real construído em miniatura. Este trem só é visível para os jogadores através de PDAs. Uma tendência, tanto para jogos eletrônicos quanto para RV, é o aparecimento de ambientes virtuais tridimensionais acessíveis pela internet, como o Second Life [23].

\subsubsection{Medicina}

$\mathrm{Na}$ área médica, as aplicações desenvolvidas exigem ambientes virtuais muito realísticos, nas quais os usuários devem ter a sensação de vivenciar a situação, ou há a necessidade da geração de sensações muito próximas às reais, com tempo de resposta e métodos implementados muito precisos.

A medicina tem se beneficiado bastante dos recursos tecnológicos oferecidos pela RV e RA nos últimos anos. Esses sistemas fornecem um recurso de ensino e treinamento muito poderoso das estruturas anatômicas. A maioria das aplicações médicas é feita com a orientação de imagens cirúrgicas. Estudos de imagens no préoperatório, como, por exemplo, tomografia computadorizada, ressonância magnética e ultrassom (sensores não invasivos), provêem ao cirurgião a visão necessária da anatomia interna do paciente e, pelo estudo dessas imagens, a cirurgia é planejada. Os sistemas também podem ser usados para o treinamento de cirurgiões novatos. Instruções virtuais também podem lembrar ao médico os passos requeridos. Entre as inúmeras vantagens das aplicações de RA na área médica, as mais visíveis dizem respeito à fidelidade das imagens coletadas, já que são feitas em tempo real, na sala de cirurgia, aumentando com isso o desempenho de toda a equipe cirúrgica e também propiciando a eliminação da necessidade dos procedimentos dolorosos e mais invasivos [15]. Muitos projetos estão sendo desenvolvidos também para suportar a cirurgia a distância.

\subsubsection{Planejamento Urbano}

A utilização de aplicações de RA e RV permite a discussão detalhada de um projeto de arquitetura em 3D antes mesmo da construção de uma maquete. Qualquer modificação no projeto é visualizada em tempo real. Dessa forma, o projeto pode ser discutido simultaneamente por arquitetos, engenheiros, construtores e quem mais estiver envolvido em seu planejamento, desenvolvimento e implantação. As aplicações nesta área incluem a exibição ou a recuperação de prédios e de outros elementos inexistentes ou em ruínas, enfatizando o planejamento urbano e a arqueologia. A grande vantagem em usar este tipo de tecnologia no planejamento das cidades é a facilidade de interação e visualização da opinião de cada usuário, bastando para isso apenas pegar e arrastar os prédios como se fossem simples caixas sobre um mapa numa mesa [19]. 


\subsubsection{Educação}

A RV e RA contribuem de maneira significativa na área da educação como processo de exploração, descoberta, observação e construção de uma nova visão do conhecimento, oferecendo ao aprendiz a oportunidade de melhor compreensão do objeto de estudo.

Essas tecnologias, portanto, têm potencial de colaborar no processo cognitivo do aprendiz, proporcionando não apenas a teoria, mas também a experimentação prática do conteúdo em questão [24]. A introdução da RA na matemática, por exemplo, pode eliminar uma das grandes dificuldades do aluno: visualizar um problema complexo de geometria. Ela fornece a professores e estudantes um método intuitivo e colaborativo de aprender. Os alunos veem os objetos tridimensionais, sem precisar imaginá-los ou desenhá-los numa folha de papel [25]. Quando envolvido e totalmente imerso no ambiente virtual, o usuário desenvolve um comportamento natural e intuitivo, procurando agir como o faria no mundo real e, através da interação, receber a resposta ideal para suas ações. Aqui, a RV e RA não são tratadas apenas como "mais uma ferramenta" para melhorar a aprendizagem, mas como um poderoso instrumento de aprendizagem. Segundo Bell e Fogler [26], a principal vantagem de utilizar a RV é a capacidade de visualizar situações e conceitos que não poderiam ser vistos de outra forma e, ainda, de imergir o aluno nessa visualização. Segundo os autores, o interesse e o entusiasmo do aluno são também evidentes benefícios da RV.

A potencialidade dessas novas tecnologias está, principalmente, no fato de permitir a exploração de alguns ambientes, processos ou objetos, não por meio de livros, fotos, filmes ou aulas, mas da manipulação e análise virtual do próprio alvo do estudo.

A introdução da RV e, consequentemente, da RA na educação demonstra um novo paradigma, que possibilita uma educação de forma dinâmica, criativa, colocando o aluno no centro dos processos de aprendizagem e buscando a formação de um ser crítico, independente e construtor de seu conhecimento. Uma grande preocupação é com o investimento em hardware e software, mas hoje em dia é possível montar uma boa plataforma com custo relativamente baixo.

\subsection{Recursos e Ferramentas da Realidade Aumentada}

Desenvolver aplicações de RA ainda é uma tarefa desafiadora, até mesmo depois de alguns anos de pesquisa, de avanço tecnológico, disponibilidade de produtos com custos acessíveis e da construção de protótipos. Vários grupos de pesquisa têm desenvolvido uma série de aplicações para RA que permitem a criação de softwares para explorar as suas possíveis interfaces. Dentre esses estão: Coterie [27], Tinmith-evo5 [21], DWARF [28], ARToolKit [29,30] e Studierstube [31, 32]. Os pesquisadores que disseminaram essas áreas, gratuitamente, através do desenvolvimento de recursos disponibilizados à sociedade tiveram, no entanto, papel fundamental. A biblioteca ARToolKit é um dos exemplos mais marcantes de recursos gratuitos e livres, mas existem vários outros disponibilizados por pesquisadores e, mais recentemente, por empresas.

O funcionamento do ARToolKit baseia-se no uso de marcadores. Permite o uso de técnicas de visualização computacional para calcular a posição da câmera e sua orientação em relação a estes marcadores, que podem ser cartões de papelão com um símbolo impresso e uma moldura quadrada, como no exemplo utilizado neste trabalho, apresentado na Figura 3.

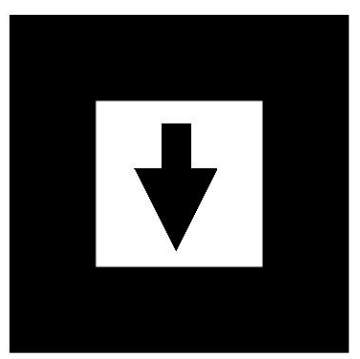

Figura 3. Marcador utilizado no presente trabalho 
O sistema sobrepõe objetos virtuais aos marcadores ou a pontos definidos pelos mesmos. A saída, portanto, nada mais é do que uma imagem formada de um ou mais objetos virtuais, animados ou estáticos, sobre o marcador ou regiões determinadas pelo mesmo. Esta biblioteca foi desenvolvida, originalmente, para servir de apoio na concepção de interfaces colaborativas pelo Dr. Hirokazu Kato, na Universidade de Osaka. Desde então, tem sido mantida pelo Human Interface Technology Laboratory (HIT Lab), da Universidade de Washington, e pelo HIT Lab NZ, da Universidade de Canterbury, em Christchurch. O ARToolKit é uma biblioteca de código aberto, escrita na linguagem $\mathrm{C}$, para concepção de aplicações em RA. O pacote inclui bibliotecas de rastreamento e disponibiliza o código fonte completo, tornando possível o transporte do código para diversas plataformas, ou a sua adaptação para resolver as especificidades de suas aplicações. O rastreamento óptico oferecido pelo ARToolKit possibilita extrair, de forma rápida, a posição e orientação de padrões marcadores apenas com o uso de um computador e uma webcam convencional.

\section{Aplicação da Realidade Aumentada}

A proposta deste artigo é apresentar o desenvolvimento de uma aplicação de RA empregando a biblioteca ARToolKit, com o intuito de incrementar o ambiente de ensino e aprendizagem para os estudantes de Arquitetura da FAU/UFRJ. Através de elementos virtuais, adicionados e associados aos modelos dos alunos, a aplicação fornece informações valiosas, como sentido, direção e posição da carga aplicada "P" e respectivas reações de apoio, além da configuração deformada de determinadas estruturas reticuladas. Essas informações, ligadas virtualmente aos modelos, são muito importantes e servem de apoio, como um complemento ao conhecimento do aluno, no processo de aprendizagem.

Em razão de a disciplina Modelagem dos Sistemas Estruturais ser a primeira na área de estruturas do citado curso de Arquitetura e Urbanismo, modelos simples de estruturas reticuladas foram concebidos para a implementação do programa. Portanto, três estruturas foram construídas (Figura 4): uma viga simplesmente apoiada, uma viga contínua, com dois vãos de mesmo comprimento, e um pórtico plano, com barras de mesmo tamanho. Novas versões do programa estão sendo desenvolvidas, pelos autores com o objetivo de analisar outros modelos de estruturas reticuladas concebidas pelos alunos.

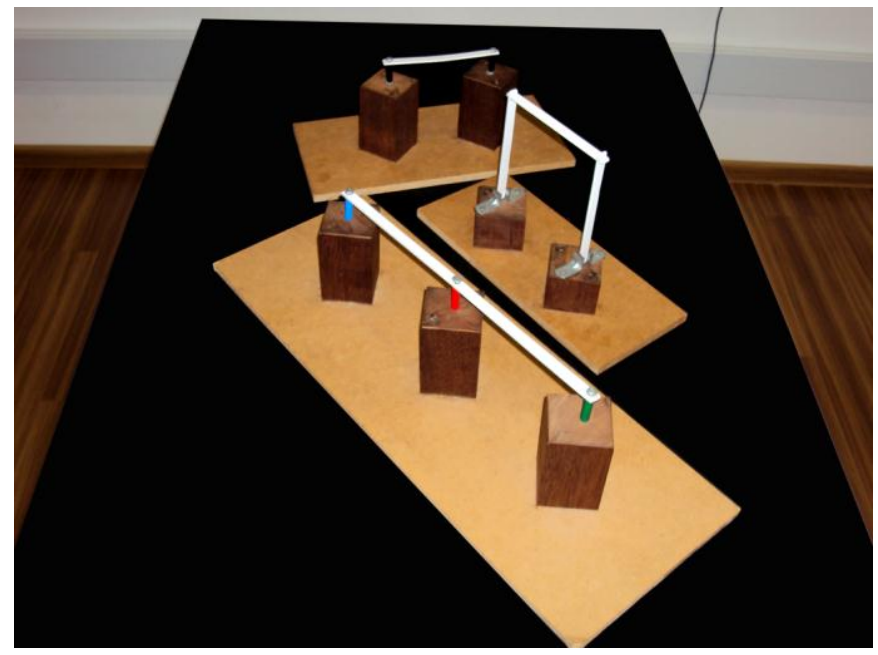

Figura 4. Modelos físicos qualitativos

As barras dos modelos reduzidos foram feitas com PVC, que se trata de um material relativamente flexível, permitindo uma melhor percepção visual dos deslocamentos da estrutura. Esses elementos foram, propositadamente, posicionados na direção de menor momento de inércia para aumentar os seus deslocamentos, facilitando a visualização da configuração deformada.

A versão utilizada do ARToolKit foi a 2.68. Para a compilação e teste desta versão foram usados os equipamentos Pentium III e Athlon XP, executando Windows XP com instalação de câmeras. O ARToolKit usou também o Vision SDK e a biblioteca GLUT de interface com o OpenGL. O código-fonte foi desenvolvido utilizando o Microsoft Visual Studio .NET 2003. A seguir, é apresentado um trecho do código. 


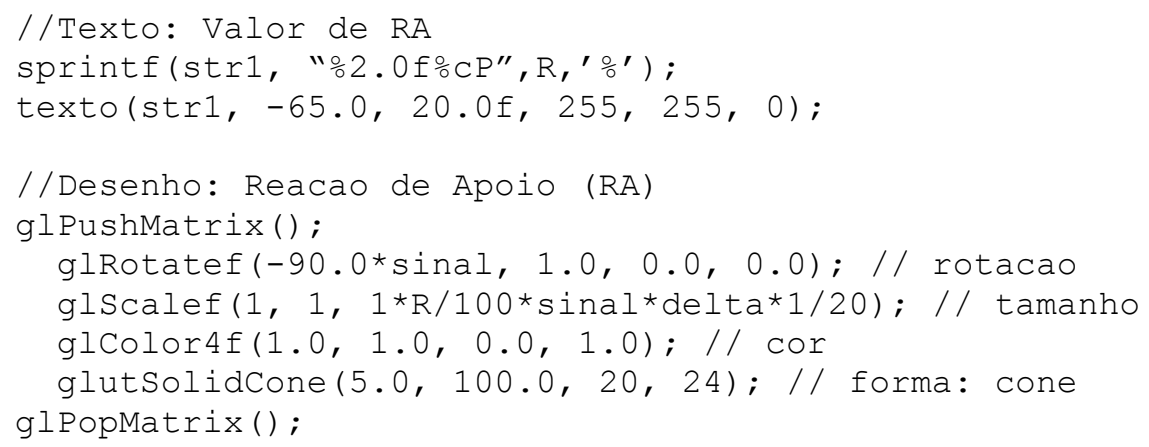

O primeiro passo no desenvolvimento da implementação foi uma produção prévia do ambiente. Uma webcam é utilizada para capturar imagens contendo o marcador impresso num cartão de papelão. O ARToolKit identifica este marcador e coloca um objeto virtual na posição do cartão. Este cartão, onde o marcador foi impresso, trata-se de um cartão suficientemente rígido para resistir ao forte manuseio. Quando está danificado, com dobras ou amassado, torna-se difícil para o programa reconhecê-lo, o que dificulta a análise. Este marcador é desenhado em tamanho adequado para se adaptar à distância da câmera. O programa foi desenvolvido para fornecer ao usuário, além da configuração deformada real da estrutura, a visualização virtual no dispositivo de vídeo da carga aplicada $\mathrm{P}$ e das reações de apoio devidas a essa carga.

Os três tipos de modelos usados pelo programa devem ser identificados durante uma fase de calibragem, na qual são definidos, por meio do marcador, pontos importantes da estrutura, como, por exemplo, a localização dos apoios. Além da configuração deformada, a carga aplicada e as reações de apoio determinadas pelo programa para o modelo da viga simplesmente apoiada estão apresentadas na Figura 5.

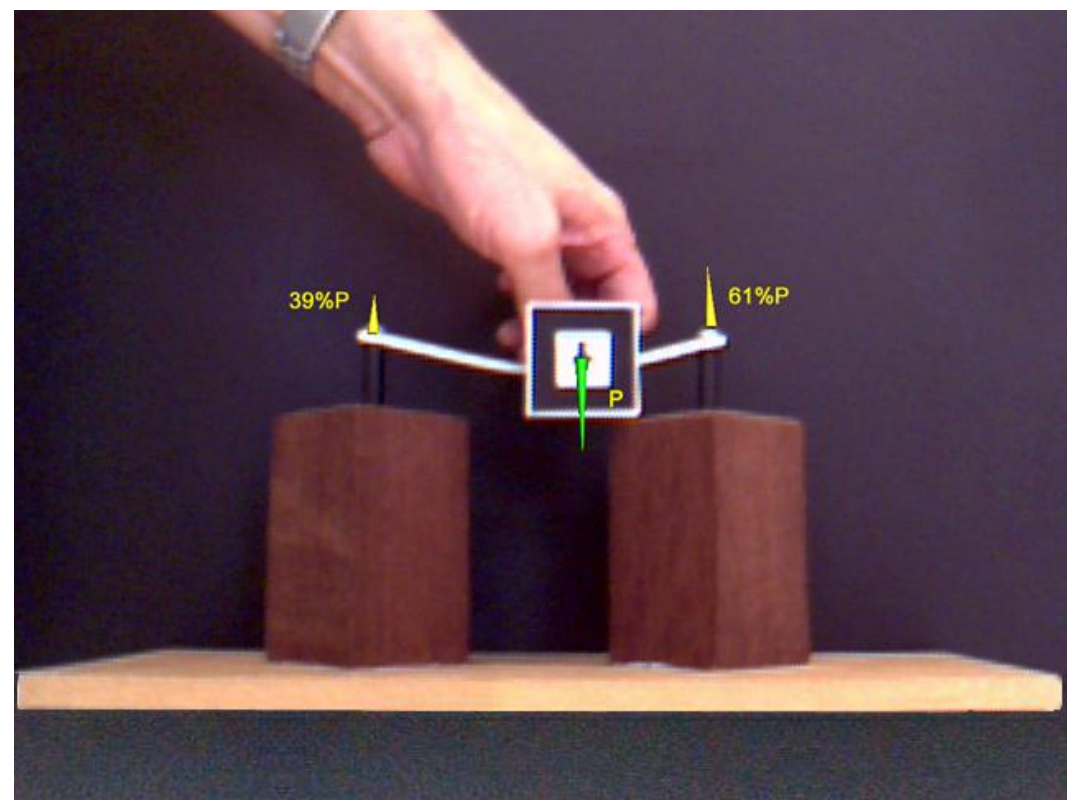

Figura 5. Reações de apoio da viga biapoiada

Com o objetivo de melhor entender o comportamento estrutural de uma viga submetida a uma carga concentrada, o aluno pressiona com os dedos a estrutura na direção vertical. Ao utilizar o programa, a webcam captura a imagem, reconhecendo o marcador, que está nas mãos do usuário. Enquanto este move o cartão, aplicando uma força, a ferramenta mostra as informações virtuais sobrepostas à viga, representando a carga aplicada "P" sua posição, e as reações de apoio. A Carga "P" e as reações de apoio mudam de comprimento, em razão da intensidade da força aplicada e à mudança de posição da mesma. Esta última altera também os valores das reações de apoio, pois, quando o ponto de aplicação da carga é modificado pelo usuário, os valores das reações de apoio também mudam, automaticamente, de acordo com as equações de equilíbrio implementadas no programa. 
As Figuras 6 e 7 mostram a carga aplicada, as reações de apoio e a configuração deformada do modelo de viga contínua e de pórtico plano, respectivamente.

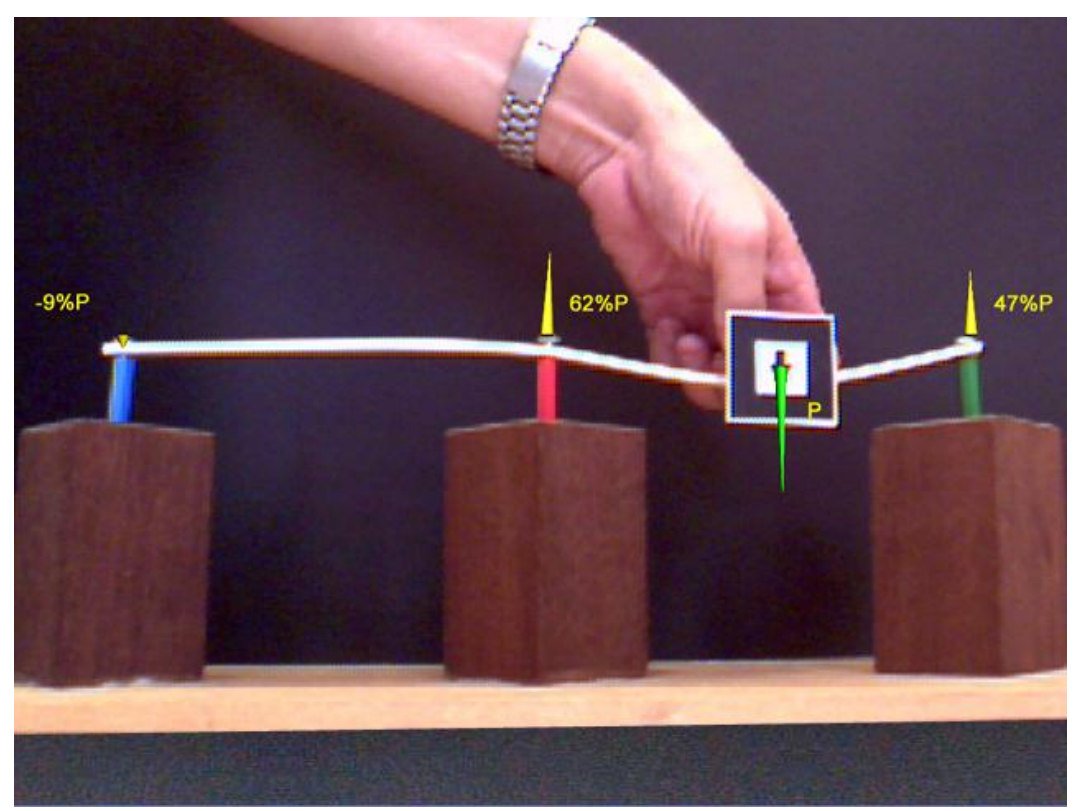

Figura 6. Reações de apoio da viga contínua

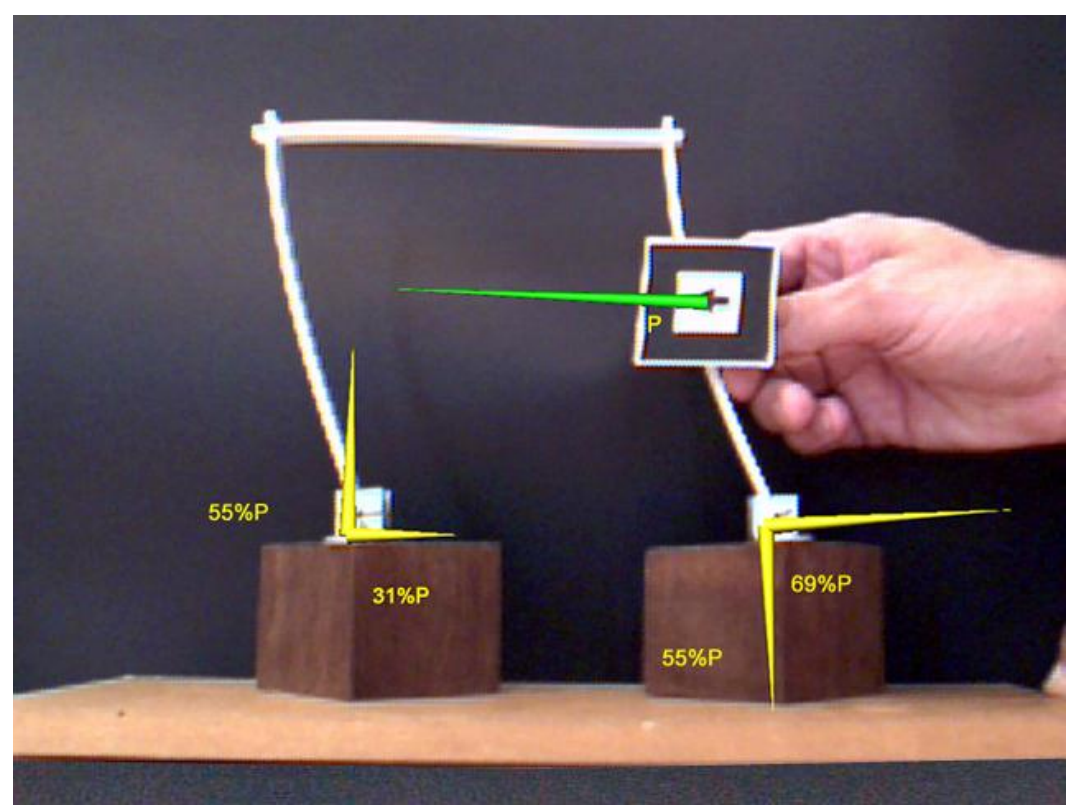

Figura 7. Reações do pórtico plano

No caso do modelo da viga contínua, além das conclusões formuladas anteriormente, o estudante pode deduzir que, quando a força é aplicada apenas num dos vãos da viga, o outro tende a se levantar e a reação do seu apoio de extremidade, distante do apoio central, muda de sentido. Portanto, o aluno pode visualizar, nitidamente, a continuidade dos deslocamentos da viga estudada e a necessidade de armação positiva no vão em que a força está aplicada, e de armação negativa no outro vão e sobre o apoio central, no caso de uma viga de concreto armado com dois vãos contínuos, onde ocorre tração. De acordo com a curvatura apresentada na configuração deformada da estrutura, o usuário pode determinar as fibras tracionadas, ou comprimidas, dos elementos.

A maior dificuldade encontrada nesta aplicação foi a instabilidade dos objetos virtuais, devida às limitações de rastreamento do algoritmo do ARToolKit. Essas limitações ocorreram, sobretudo, por causa da luz 
do ambiente, do tipo de câmera usado, do ângulo e da distância entre a câmera e o marcador. No presente trabalho, dois tipos de câmera foram utilizados: SoC PC-Camera e LifeCam VX-6000. Esta instabilidade despertou nos autores o interesse por uma pesquisa análoga na FAU/UFRJ, utilizando a RV (Figura 8), com o intuito de realizar uma comparação futura entre as duas técnicas aplicadas ao ensino da disciplina Modelagem de Sistemas Estruturais.

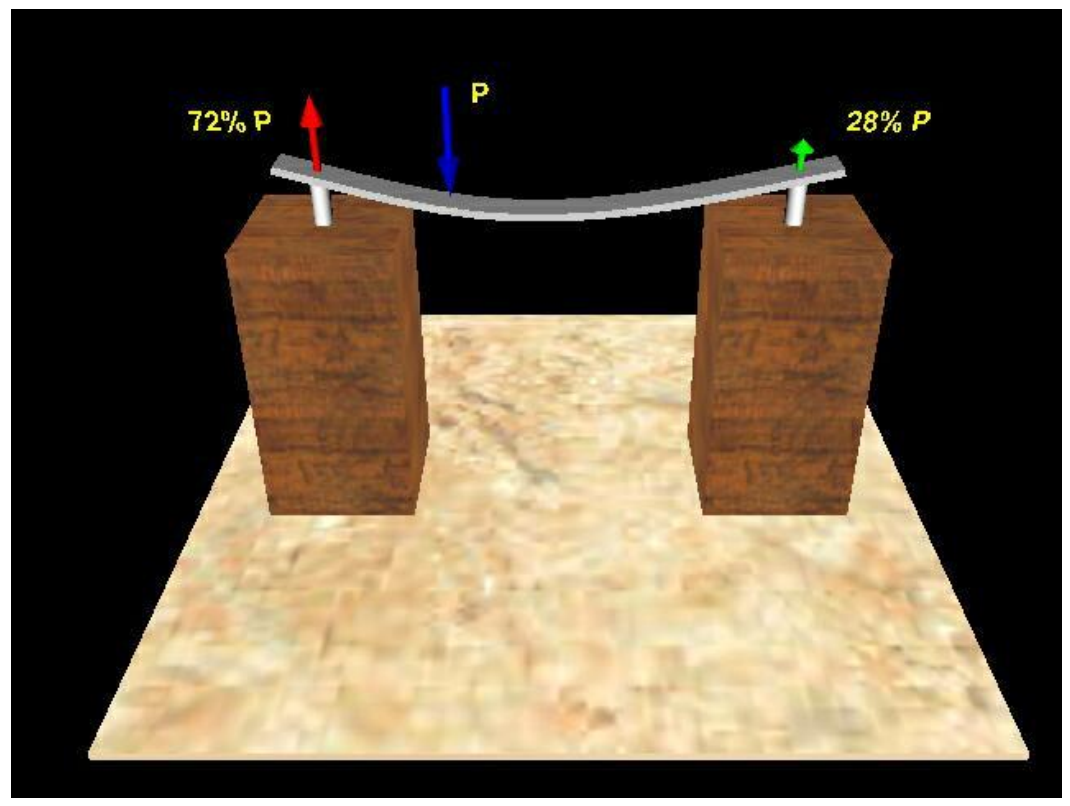

Figura 8. Aplicação em RV

A aplicação dessa ferramenta possibilita ao aluno de arquitetura desenvolver a sua capacidade de observação e cultivar seu sentimento, percepção e intuição para o entendimento do comportamento estrutural, de forma qualitativa, incrementado pelas seguintes conclusões:

• Quanto maior a carga aplicada "P”, maiores são os deslocamentos.

- Enquanto a carga "P" cresce, as reações de apoio também crescem proporcionalmente.

- A soma das reações de apoio na direção da força aplicada é igual à carga aplicada, e na outra direção é nula, ou seja, o sistema está em equilíbrio.

- Quanto mais próxima estiver a carga aplicada de um apoio, maior é a reação, na direção desta carga, neste apoio.

- Na viga contínua com dois vãos, quando a força é aplicada apenas num dos vãos, o outro tende a se levantar, mudando o sentido da reação do apoio distante do apoio central. Este fato é percebido pelo sinal negativo da reação.

- As fibras tracionadas e comprimidas da viga são facilmente visualizadas, por meio das curvaturas apresentadas na configuração deformada.

- No caso do pórtico plano, o aluno pode notar a tendência de tombamento da estrutura e as reações de apoio contrárias a este movimento.

- Os pórticos das edificações, na maioria das vezes, consistem de vigas ligadas às colunas por meio de ligações consideradas rígidas, de forma que a estrutura, como um todo, torna-se mais resistente aos efeitos de cargas laterais devidas ao vento e a terremotos, ou seja, o ângulo formado pelos elementos estruturais nas conexões após a ação da carga aplicada mantém-se constante. Este fato é visível através da sua configuração deformada.

A Figura 9 apresenta alguns alunos interagindo com o programa em sala de aula. 


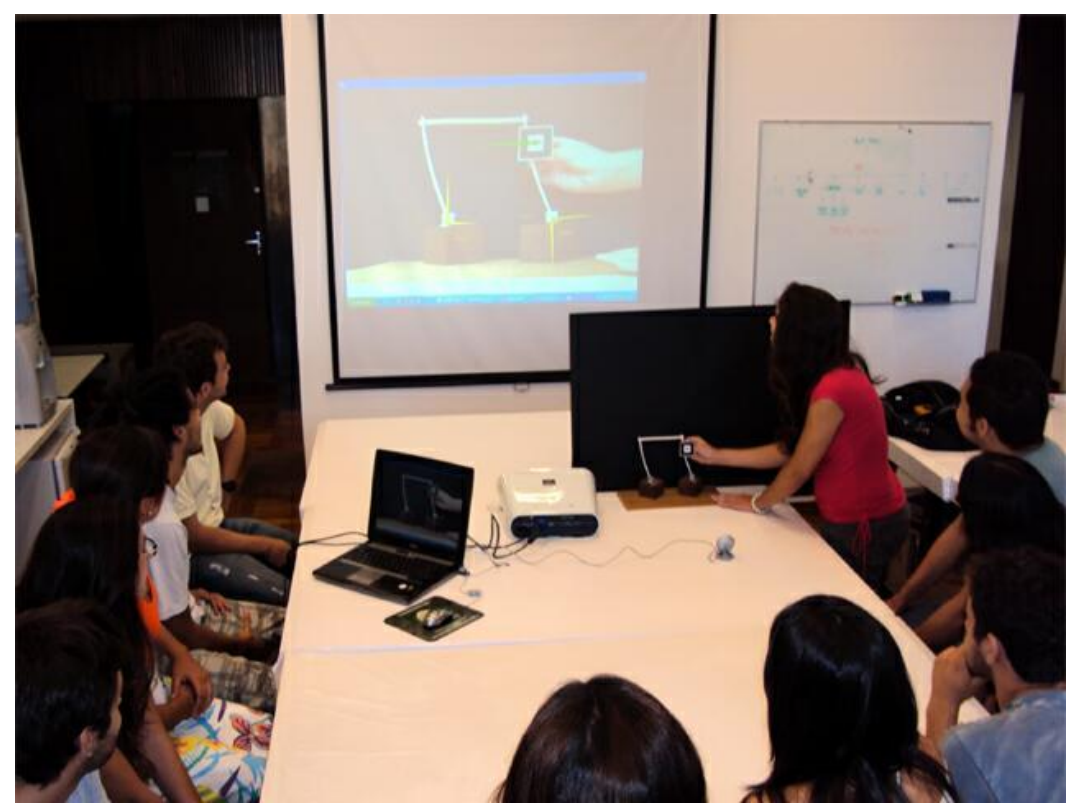

Figura 9. Alunos interagindo com o programa de RA

\section{Conclusões}

A introdução à análise estrutural na Faculdade de Arquitetura e Urbanismo da Universidade Federal do Rio de Janeiro é, inevitavelmente, feita pela análise do comportamento de modelos físicos qualitativos e, como mostrado neste trabalho, foi incrementada com a aplicação de técnicas de Realidade Aumentada (RA). Esse procedimento possibilitou aos alunos uma melhor percepção da configuração deformada de sistemas estruturais, de maneira qualitativa, proporcionando a ampliação do conhecimento das respostas desses sistemas quando submetidos a uma carga concentrada em diferentes pontos.

Este artigo apresentou o desenvolvimento de uma aplicação de RA utilizando a biblioteca ARToolKit, com o objetivo de melhorar o ambiente de ensino e aprendizagem principalmente para os estudantes do curso de Arquitetura e Urbanismo da FAU/UFRJ.

A compreensão do comportamento estrutural por parte dos alunos calouros, atualmente, com a aplicação da RA, pode ser alcançada de maneira mais concreta, reforçada, consolidada e incrementada por possíveis constatações. Numa experimentação informal, os estudantes de Arquitetura da FAU/UFRJ demonstraram o desejo de utilizar ferramentas e técnicas computacionais modernas no processo de ensino e aprendizagem e, desde então, pesquisas neste campo vêm sendo conduzidas pelo Departamento de Estruturas, empregando técnicas da Realidade Virtual e da Realidade Aumentada. Essa iniciativa foi bem aceita pelos alunos, que começaram a fornecer sugestões valiosas para melhorias, reconhecendo que a utilização de ferramentas deste tipo trata-se de um incremento importante e necessário no curso de Arquitetura e Urbanismo FAU/UFRJ.

Finalmente, como desenvolvimento futuro, uma nova versão do programa está em fase de elaboração, visando ao refinamento e à otimização do código do programa, que resultará numa ferramenta mais ampla, contemplando modelos de estruturas mais complexas, incluindo novos modelos que serão criados pelos alunos, também, para efeitos de comparação, uma versão análoga a este programa, aplicando técnicas de RV, está sendo desenvolvida. Ainda, um estudo observacional está previsto na FAU/UFRJ para avaliar os benefícios da utilização desta aplicação, bem como compará-la com a ferramenta análoga em RV, que se encontra em fase de desenvolvimento.

\section{Referências}

[1] SAlVADORI, M.; HELlER, R. Structure in Architecture, New Jersey: Prentice Hall, 3rd edition, 1963. 
[2] POLILlO, A. Considerações Sobre o Ensino de Estruturas nos Cursos de Formação de Arquitetos. Rio de Janeiro: Sedegra-Rio, 1968.

[3] GORDON, J. E. Structures or Why Things Don’t Fall Down. London: Da Capo Press, 1978.

[4] SAlVADORI, M. G.; TEMPEL, M. Architecture and Engineering: An Illustrated Teacher's Manual On Why Buildings Stand Up. New York: Salvadori Educational Center On The Built Environment, 1983.

[5] SANTOS, J. A. Sobre a Concepção, o Projeto, a Execução e a Utilização de Modelos Físicos Qualitativos na Engenharia de Estruturas. 1983. Tese (Mestrado em Engenharia Civil) - Escola Politécnica da Universidade de São Paulo, São Paulo.

[6] HILSON, B. Basic Structural Behaviour - Understanding Structures From Models. London: Thomas Telford, 1993.

[7] RODRIGUES, P. F. N.; HERMIDA A. S. Modelagem de Elementos Básicos de Estruturas para a Análise Qualitativa do Comportamento Estrutural. Revista de Ciência \& Tecnologia, Nova Iguaçu, v. 6, n. 1, p. 19-28, 2006.

[8] ABDELMAWLA, S.; ELNIMEIRI, M.; KRAWCZYK, R. Structural Gizmos. In: ETERNITY, INFINITY AND VIRTUALITY IN ARCHITECTURE - 22ND ANNUAL CONFERENCE OF THE ASSOCIATION FOR COMPUTER-AIDED DESIGN IN ARCHITECTURE (ACADIA 2000), 2000, Washington, USA. Proceedings. .. Washington D.C., 2000. p. 115-121.

[9] PICCOLOTTO, M.; RIO, O. Structural Design Education With Computers. In: 1995 NATIONAL CONFERENCE OF THE ASSOCIATION FOR COMPUTER-AIDED DESIGN IN ARCHITECTURE. (ACADIA 95), 1995, Washington, USA. Proceedings. . . Seattle: University of Washington, 1995, p. 285 299.

[10] VASSIGH, S. Structures E-Book. ACADIA Quarterly, Chicago: The School of the Art Institute of Chicago, v. 18, n. 3, p. 14-15, 1999.

[11] GUIDERA, S. G. Exploring the architecture of structure: Integrating structures into design studio using object-oriented CAD. Proceedings of the 2003 ASEE Annual Conference \& Exposition (online), Nashville, USA, 2003. Disponível em: <http://soa.asee.org/paper/conference/paper-view.cfm?id=18586>. Acesso em: 08 jun. 2009.

[12] KIM, I. Y.; DE WECK, O. L.; NADIR, W.; YOUNG, P.; WALLACE, D. Innovative Modern Engineering Design and Rapid Prototyping Course: A Rewarding CAD/CAE/CAM Experience for Undergraduates. Proceedings of the $\mathbf{2 0 0 4}$ American Society For Engineering Education (ASEE) Annual Conference \& Exposition (online). Salt Lake City, Utah, USA, session 332, 2004. Disponível em:

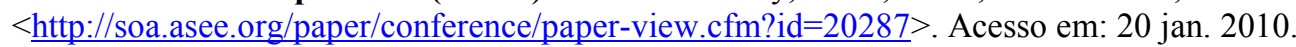

[13] MILLARD, S. G. Computer Aided Learning (CAL) and Computer Aided Assessment (CAA) in Civil Engineering. Proceedings of the 3rd International CDIO (Conceiving - Designing Implementing - Operating) Conference (online), Massachusetts, USA, 2007. Disponível em:

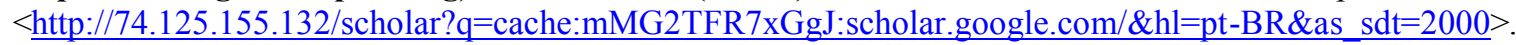
Acesso em: 09 jan. 2009.

[14] MSE: Modelagem dos Sistemas Estruturais. Site da disciplina da Faculdade de Arquitetura e Urbanismo da

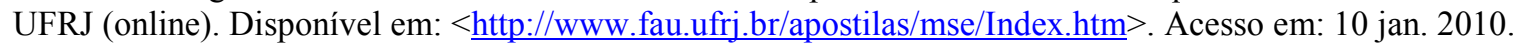

[15] AZUMA, R. T.; BAILlOT, Y.; BEHRINGER, R.; FEINER, S.; JULIER, S.; MACINTYRE, B. Recent Advances in Augmented Reality. IEEE Computer Graphics and Applications, v. 21, n. 6, p. 34-47, 2001. 
[16] AZUMA, R. T. A Survey of Augmented Reality, Presence: Teleoperators and Virtual Environments, v. 6, n. 4, p. 355-385, 1997.

[17] GRIMSON, W., ETTINGER, G., KAPUR, T., LEVENTON, M., WELLS, W., KIKINIS, R., Utilizing Segmented MRI Data in Image-guided Surgery. International Journal of Pattern Recognition and Artificial Intelligence, v. 11, n. 8, pp. 1367-1397, 1997.

[18] SISCOUTTO, R. A., RAPOSO, A. B., TORI, R., SZENBERG, F., CELES, W., GATTASS, M. Estereoscopia. In: FUNDAMENTOS E TECNOLOGIA DE REALIDADE VIRTUAL E AUMENTADA, 2006, Porto Alegre, Brasil. Proceedings. .. v. 1, Editora SBC - Sociedade Brasileira de Computação, 2006, p. 221-245.

[19] BASTOS, N. C., Arquitetura para Dispositivos Não-Convencionais de Interação Utilizando Realidade Aumentada: Um Estudo de Caso. 2005. Trabalho de Conclusão de Curso (Graduação em Ciência da Computação) - Universidade Federal de Pernambuco, Recife.

[20] PAVLOVIC, V.I., SHARMA, R., HUANG, T.S. Visual Interpretation of Hand Gestures for HumanComputer Interaction: A Review. IEEE Transactions on Pattern Analysis and Machine Intelligence, v. 19, n. 7, p. 677-695, 1997.

[21] PIEKARSKI, W., THOMAS, B. ARQuake: The Outdoor Augmented Reality Gaming System. Communications of the ACM, v. 45, n. 1, p. 36-38, 2002.

[22] WAGNER, D., PINTARIC, T., LEDERMANN, F., SCHMALSTIEG, D. Towards Massively Multi-User Augmented Reality on Handheld Devices. In: INTERNATIONAL CONFERENCE ON PERVASIVE COMPUTING, 2005, Munich, Germany. Proceedings... Munich, 2005, p. 208-219.

[23] SECOND LIFE. Second Life: Your World. Your Imagination. Disponível em: $<$ http://secondlife.com>, Acesso em: 05 out. 2009.

[24] CARDOSO, A., LAMOUNIER, E. A. Aplicações na Educação e Treinamento. In: REALIDADE VIRTUAL E AUMENTADA - UMA ABORDAGEM TECNOLÓGICA, 2008, Porto Alegre, Brasil. Proceedings... v. 1, Editora SBC - Sociedade Brasileira de Computação, 2008, p. 343-357.

[25] LOPES, L. F. B. O Estudo e a Implementação de Interfaces para Utilização em Sistemas de Realidade Aumentada, 2005. Tese (Mestrado em Ciência da Computação) - Centro Universitário Eurípides de Marília, Fundação de Ensino Eurípides Soares da Rocha, Marília, São Paulo.

[26] BELL, J. T., FOGLER, H. S. The Investigation and Application of Virtual Reality as an Educational Tool. In: THE AMERICAN SOCIETY FOR ENGINEERING EDUCATION ANNUAL CONFERENCE, 1995, Anheim, USA. p. 1718-1728, Anheim, 1995, p. 1718-1728.

[27] BUTZ, A., HÖLLERER, T., FEINER, S., MACINTYRE, B., BESHERS, C. Enveloping Users and Computers in a Collaborative 3D Augmented Reality. In: INTERNATIONAL WORKSHOP ON AUGMENTED REALITY (IWAR '99), 1999, San Francisco, USA. Proceedings. . . San Francisco, CA, USA, 1999, p. 35-44.

[28] BAUER, M., Hilliges, A., MACWIlliams, A., SANDOR, C., WAGNER, G., KLinKer, G., NEWMAN, J., REITMAYR, G., PINTARIC, T., FAHMY, T., SCHALSTIEG, D. Integrating Studierstube and DWARF. In: INTERNATIONAL WORKSHOP ON SOFTWARE TECHNOLOGY FOR AUGMENTED REALITY SYSTEMS, 2003, Tokyo, Japan. Proceedings. .. Tokyo, 2003, p. 1-5.

[29] KATO, H. ARToolKit. Human Interface Technology Laboratory, University of Washington, DC., USA. Disponível em: <www.hitl.washington.edu/artoolkit $>$. Acesso em: 02 mai. 2009. 
[30] KATO, H., \& BILLINGHURST, M. Marker Tracking and HMD Calibration for a Video-based Augmented Reality Conferencing System. In: INTERNATIONAL WORKSHOP ON AUGMENTED REALITY (IWAR '99), 1999, San Francisco, USA. Proceedings. .. San Francisco, CA, USA, 1999, p. 85-94.

[31] FUHRMANN, A., PURGATHOFER, W. Studierstube: An Application Environment for Multi-User Games in Virtual Reality. In: JAHRESTAGUNG DER GESELLSCHAFT FÜR INFORMATIK, 2001, Viena, Austria. Proceedings. . Viena, 2001, p. 1185-1190.

[32] SCHMALSTIEG, D. Studierstube: Augmented Reality Project. Disponível em: <www.studierstube.org>. Acesso em: 05 out. 2009. 